\title{
Floating-gate MOS structures and applications
}

\begin{abstract}
Floating-gate MOS transistor (FGMOS) has proved to be suitable for low-voltage analog applications, owing to its threshold voltage programmability. This tutorial paper presents FGMOS based circuit structures and their applications in analog signal processing. The FGMOS based current mirror and its application as voltage controlled current source has been presented. The performance of these structures has been verified using PSpice simulations for 0.5 im CMOS technology at $0.75 \mathrm{~V}$.
\end{abstract}

Keyword: Current mirror; Floating-gate MOS transistor; Low-voltage circuit 